\title{
Berechnung einiger Wirkungsquerschnitte am Beryllium
}

\author{
Von Friedrich SCHLÖGL* \\ Aus dem Institut für physikalische Chemie der Universität Göttingen \\ (Z. Naturforschy. 3 a, 229-240 [1948]; eingegangen am 13. Oktober 1947)
}

\begin{abstract}
Es wird für den Berylliumkern ein vereinfachtes Zweikörpermodell entwickelt, an dem die Wirkungsquerschnitte für den Kernphotoeffekt, die Neutronenstreuung und den $(n, 2 n)$-Prozeß mit Hilfe einer Störungsrechnung bis zu maximalen Einstrahlungsenergien von einigen $\mathrm{MeV}$ berechnet und mit empirischen Daten verglichen werden.
\end{abstract}

$\mathrm{D}$ er Kern des gewöhnlichen Berylliums ${ }^{9} \mathrm{Be}$ ist von allen in der Natur vorkommenden stabilen Kernen derjenige, für den die Abtrennungsarbeit eines Neutrons am kleinsten ist. Sie ergibt sich aus dem Massendefekt als $\varepsilon=1,67 \mathrm{MeV}^{1}$. Entsprechend dieser Tatsache sollen für die folgenden Rechnungen alle Bausteine des ${ }^{8} \mathrm{Be}$ zu einem Kernrumpf zusammengefaßt werden, um den sich, relativ locker gebunden, ein Neutron bewegt. Dieser Rumpf besteht aus den Bausteinen zweier $\alpha$-Teilchen, und man wird ihm den Spin Null zuordnen. Nun ist ${ }^{8} \mathrm{Be}$ nicht stabil und zerfällt nach einer nicht genau bekannten, sehr kurzen Lebensdauer in zwei $\alpha$-Teilchen. Für die Zerfallsenergie läßt sich nach der Massenbilanz und direkteren Messungen von $\mathrm{L}$ a a f $\mathrm{f}^{2}$ nur eine obere Grenze angeben. Nach dem Ga m o w schen Kernmodell ergibt sich dann eine untere Grenze für die Lebensdauer bei $10^{-9}$ sec. Die im folgenden betrachteten Kernreaktionen erfolgen aber in wesentlich kürzeren Zeiten, so daß dafür das Zweikörpermodell zulässig ist, das mit einem kompakten Kernrumpf rechnet. Das Kernmoment des ${ }^{9}$ Be ist wahrscheinlich ${ }^{3} / 2$; allerdings ist der Wert $1 / 2$ nicht ganz ausgeschlossen ${ }^{3}$. Der Grundzustand ist wahrscheinlich ein P-Zustand, obwohl ein S-Zustand noch möglich wäre ${ }^{4}$.

\section{Der Kernphotoeffekt}

Beim Kernphotoeffekt wird ein Neutron unter Einwirkung einer $\gamma$-Strahlung aus dem Kern emittiert. Er läßt sich nach dem entwickelten Zweikörpermodell folgendermaßen verstehen. Im Kern bewegen sich Rumpf und Neutron um den gemeinsamen Schwerpunkt. Der Rumpf trägt die

\footnotetext{
* Gekürzte Göttinger Dissertation, vgl. die Bemerkung am Schluß.

1 Berechnet nach J. M a t t a u ch u. S. F l üg g e, Kernphysikal. Tabellen, Berlin 1942.
}

gesamte elektrische Kernladung und tritt in Wechselwirkung mit dem elektrischen Feld der einfallenden $\gamma$-Strahlung. Diese kann zur Abtrennung des Neutrons führen. Der Effekt ist ein rein photoelektrischei. Ein photomagnetischer könnte stattfinden, wenn der Rumpf ein Spinmoment besäße und seine Wechselwirkung mit dem Neutron von der gegenseitigen Spinstellung abhinge.

Die Wechselwirkung mit der Strahlung sei als Störung behandelt. Die Schrödinger-Gleichung des ungestörten Kernsystems läßt sich nach Schwerpunkts- und Relativkoordinate separieren. Die Schwerpunktsbewegung ist eine einfache Translation. In der Relativkoordinate $\mathfrak{x}$ des Neutrons gegenüber dem Rumpf gilt die SchrödingerGleichung

$$
-\frac{\hbar^{2}}{2 M} \Delta u+[V(r)-E] u=0 .
$$

Dabei ist $M$ die reduzierte Masse des Systems, $u(\mathfrak{r})$ die. Zustandsfunktion, und es ist dabei angenommen, daß sich die Wechselwirkung zwischen Rumpf und Neutron durch eine Potentialfunktion $V(r)$ des gemeinsamen Abstandes $r$ beschreiben läßt.

Solange die $\gamma$-Energie in der Größenordnung von $m c^{2}$ liegt, ist das Verhältnis der Kerndimensionen zur Wellenlänge der Strahlung von der Größenordnung der Feinstrukturkonstanten. Man kann dann das Strahlungsfeld als ortsunabhängiges Wechselfeld beschreiben. Das heißt, man vernachlässigt den Impuls des $\gamma$-Quants, so daß in dieser Näherung die Bewegung des Kernschwerpunktes unverändert bleibt, der ohne Einschränkung als ruhend angenommen werden

2 O. L a a f f, Ann. Physik 32, 743 [1938].

3 Vgl. W. P a u l, Z. Physik 117, 774 [1941].

${ }_{4}$ Vgl. z. B. H. K o p f e r m a n n, Kernmomente, Leipzig 1940. 
kann. Dann ist $-\mathfrak{r} M / M_{R}$ die Absolutkoordinate des Kernrumpfes der Masse $M_{R}$. Ist $F \sin \omega t$ die elektrische Feldstärke der Strahlung, die in der $z$-Richtung polarisiert sei, so ist die Störenergie

$$
-z \frac{M}{M_{R}} Z e F \sin \omega t
$$

wobei $Z e$ die Kernladung sei.

Gefragt wird nach dem Übergang des ungestörten Systems vom Grundzustand mit der Lösung $u_{0}(\mathfrak{r})$ von (1) unter Einwirkung der Störung zu einem Endzustand des Kontinuums, der durch diejenige Lösung $\xi(\mathfrak{f}, \mathfrak{r})$ beschrieben wird, die für große Abstände $r$ asymptotisch in die ebene Welle $\exp (i \mathfrak{f} \mathfrak{r})$ übergeht. Zum Anfangszustand gehört die Energie $-\varepsilon$, zum Endzustand $E=\hbar^{2} k^{2} / 2 M$. Nach dem Energiesatz gilt $E=\hbar \omega-\varepsilon$. Für das Folgende soll $u_{0}$ auf 1 normiert sein. Nach dem Diracschen Störungsverfahren ergibt sich die Wahrscheinlichkeit, daß in der Zeiteinheit ein solcher Übergang mit beliebiger Ausstrahlrichtung $\mathfrak{f}$ erfolgt, zu

$$
w=4 \pi^{2}(Z e F)^{2}\left(M / M_{R}\right)^{2} \frac{M k}{h^{3}} \overline{\mid z_{0 e}{ }^{2}} .
$$

Dabei ist

$$
z_{0 e}=\int \xi^{*} z u_{0} d \mathbf{r}
$$

und der Querstrich bedeutet Mittelwertsbildung über alle Richtungen von $\mathfrak{f}$. Dèr Wirkungsquerschnitt $\sigma_{\gamma}$ für den Kernphotoeffekt ist das Verhältnis der sekundlich von einem Kern im statistischén Mittel absorbierten Energie $\hbar \omega w$ zur Energiestromdichte $c F^{2} / 8 \pi$ der einfallenden Strahlung:

$$
\sigma_{\gamma}=2\left(Z M / M_{R}\right)^{2} \frac{e^{2}}{\hbar c}\left(\gamma^{2}+k^{2}\right) l_{i} \cdot \overline{\left.z_{0 e}\right|^{2}} .
$$

Hierbei ist

$$
\gamma^{2}=\frac{2 M \varepsilon}{\hbar^{2}}
$$

Zweckmäßig wird 气 nach Kugelfunktionen des Winkels $\propto$ zwischen $\mathfrak{r}$ und $\mathfrak{f}$ entwickelt:

$$
\xi(\mathfrak{f}, \mathfrak{r})=\sum_{l=0}^{\infty} R_{l}(r) P_{l}(\cos \alpha) .
$$

Dann ergeben sich nach (3) die gewöhnlichen Auswahlregeln für Dipolstrahlung:

$$
l=l_{0} \pm 1, \quad m_{0}=0,
$$

wobei $l_{0}$ die Quantenzahl des Bahndrehimpulses im Grundzustand ist, $m_{0}$ die der $\boldsymbol{z}$-Komponente.

\section{1. $P-\mathrm{Zustand}$}

Wird der Grundzustand als ein P-Zustand angenommen, dann tragen also nur die Komponenten $l=0,2$ bei. Nun haben die Komponenten $l$ in (6) dann keine wesentlichen Werte in unmittelbarer Kernnähe, wenn $k^{2} \ll l(l+1)$ ist. Für genügend kleine Energien ist deshalb $l=2$ gegen $l=0 \mathrm{zu}$ vernachlässigen, und es wird

$$
z_{0 e}=\int R_{0}^{*} z u_{0} d \mathfrak{r} .
$$

Von den drei Möglichkeiten $m_{0}=0, \pm 1$ liefert entsprechend der zweiten Auswahlregel nur $m_{0}=0$ nicht verschwindende Matrixelemente $z_{0 e}$. Im statistischen Mittel wird deshalb

$$
\overline{z_{0 e}{ }^{2}}=\frac{1}{3} \overline{z_{0 e}^{0}{ }^{2}},
$$

wobei $z_{0 e}^{0}$ das Matrixelement für $m_{0}=0$ bedeuten soll, wofür $u_{0}$ die Gestalt $u_{0}(\mathfrak{r})=v(r) \cos \vartheta$ hat, wenn $z=r \cos \vartheta$ ist.

Wird

$$
z_{0}^{0} e=\frac{4 \pi}{3} \int_{0}^{\infty} R_{0}^{*} v r^{3} d r .
$$

geschrieben, so geht $\chi(k, r)$ für große $r$ asymptotisch in $\sin (k r+\delta)$ über, wie der Vergleich mit der Entwicklung

$\exp (i \mathfrak{f} \mathfrak{r})$

$$
=\sum_{l=0}^{\infty}(2 l+1) i^{l}(\pi / 2 k r)^{1 / 2} J_{l+1 / 2}(k r) P_{l}(\cos a)
$$

der ebenen Welle und das asymptotische Verhalten der Bessel-Funktionen $J$ zeigt.

Im folgenden soll als Längeneirheit immer ein Kernradius a gewählt werden. Beim Übergang zu anderen Einheiten ist der Wirkungsquerschnitt dann mit $a^{2}$ zu multiplizieren.

Die Wechselwirkung zwischen Kernrumpf und Neutron soll durch den einfachen Potentialansatz

$$
V(r)=\left\{\begin{aligned}
-A & \text { für } r<1 \\
0 & \text { für } r>1
\end{aligned}\right.
$$

beschrieben werden. $A$ soll so bestimmt werden, daß $-\varepsilon$ der Energieeigenwert zum Grundzustand $u_{0}$ ist. Unter Beachtung der Randbedingungen, daß $r v$ und $\chi$ für $r=0$ und $r=\infty$ verschwinden müssen, ergeben sich dann nach (1) die Lösungen 
$\approx(k, r)= \begin{cases}K \sin \lambda r & \text { für } r<1 \\ \sin (k r+\delta) & \text { für } r>1,\end{cases}$

$v v(r)= \begin{cases}N_{1}\left(\frac{\sin \varkappa r}{\% r}-\cos \% r\right) \quad \text { für } r<1 & \\ N_{2} \exp (-\gamma r)\left(1+\frac{1}{\gamma r}\right) & \text { für } r>1,\end{cases}$

wobei

$$
\begin{aligned}
& \lambda^{2}=(A+\hbar \omega) \frac{2 M}{\hbar^{2}}=\frac{A}{\varepsilon} \gamma^{2}+k^{2}, \\
& \varkappa^{2}=(A-\varepsilon) \frac{2 M}{\hbar^{2}}=\frac{A}{\varepsilon} \gamma^{2}-\gamma^{2} .
\end{aligned}
$$

Die Bedingungen der Stetigkeit der Lösungen sowie ihrer ersten Ableitungen bei $r=1$ ergeben

$$
\begin{aligned}
K & =\frac{\sin (k+\delta)}{\sin \lambda}, \\
\frac{N_{2}}{N_{1}} & =\frac{\exp (\gamma)}{1+1 / \gamma}\left(\frac{\sin \%}{\%}-\cos \varkappa\right)
\end{aligned}
$$

und liefern die Bestimmungsgleichungen für $\delta$ und $\%($ d. h. für $A)$ :

$$
\begin{gathered}
\frac{\operatorname{tg}(k+\delta)}{k}=\frac{\operatorname{tg} \lambda}{\lambda}, \\
\frac{x}{\operatorname{tg} \varkappa}=1+\frac{1+\gamma^{\prime}}{\gamma^{2}} \varkappa^{2} .
\end{gathered}
$$

ist dabei die kleinste positive Lösung der letzten Gleichung. Wie eine graphische Konstruktion zeigt, liegt sie zwischen $\pi$ und $3 \pi / 2$. Die Normierungsbedingung für $u_{0}$ heißt

$$
\int_{0}^{\infty} v^{2} r^{2} d r=\frac{3}{4 \pi}
$$

und liefert eine zweite Gleichung für $N_{1}, N_{2}$. Dabei wird zweckmäßig beachtet, daß für $r<1$ gilt:

$$
\begin{aligned}
& \sqrt{r} v(r)=N_{1} \sqrt{\pi \% / 2} J_{3 / 2}(\% r), \\
& \int_{0}^{1} r\left[J_{3 / 2}(\varkappa r)\right]^{2} d r=\frac{1}{2}\left\{\left[J_{3 / 2}(\varkappa)\right]^{2}-J_{1 / 2}(\varkappa) J_{5 / 2}(\varkappa)\right\} \\
& =\frac{1}{\pi x}\left\{1+\frac{\sin x}{\psi}\left(\cos \%-2 \frac{\sin x}{\psi}\right)\right\} \text {. }
\end{aligned}
$$

Die Integration für $r>1$ verläuft elementar. Es wird so erhalten:

$$
\begin{aligned}
& \frac{N_{1}^{2}}{2}\left\{1+\frac{\sin \psi}{\varkappa}\left(\cos \psi-2 \frac{\sin \psi}{\varkappa}\right)\right\} \\
& \quad+\frac{N_{2}^{2}}{2 \gamma}(1+2 / \gamma) \exp (-2 \gamma)=\frac{3}{4 \pi} .
\end{aligned}
$$

So sind schließlich die Eigenfunktionen (8) bestimmt.
Mit ihnen wird nach (7)

$$
\begin{aligned}
z_{0 e}^{0}= & \frac{4 \pi}{3} \frac{\exp (-i \delta)}{k} \\
& \cdot\left[N_{1} \frac{K \int_{0}^{1} r \sin \lambda r\left(\frac{\sin \varkappa r}{\varkappa r}-\cos \varkappa r\right) d r}{}\right. \\
& \left.+N_{2} \int_{1}^{\infty} r \sin (k r+\delta)(1+1 / \gamma) \exp (-\gamma r) d r\right] .
\end{aligned}
$$

Die Auswertung der Integrale erfolgt am einfachsten, indem man sie als Realteil eines komplexen Ausdruckes auffaßt und für diesen die Integration ausführt. Nach einigen Umformungen, bei denen man (10), (11) und (13) zu berücksichtigen hat, findet man

$$
\begin{gathered}
z_{0 e}^{0}=\frac{4 \pi}{3} \frac{\lambda}{k} \frac{K \exp (-i \delta)}{\gamma^{2}+k^{2}} \\
\cdot\left\{N _ { 1 } \left[\left(\sin \psi-\frac{2 \varkappa}{\gamma^{2}+k^{2}} \cos x\right) \frac{\varkappa}{\lambda} \sin \lambda\right.\right. \\
\left.+\left(\cos \psi+\frac{3-\lambda^{2} / \varkappa^{2}}{\gamma^{2}+k^{2}} \varkappa \sin x\right) \cos \lambda\right]+N_{2} \exp (-\gamma) \\
\left.\cdot\left[\left(1+\gamma+\frac{2 \gamma^{2}}{\gamma^{2}+k^{2}}\right) \frac{\cos \lambda}{\gamma}+\left(\gamma+\frac{2 \gamma^{2}}{\gamma^{2}+k^{2}}\right) \frac{\sin \lambda}{\lambda}\right]\right\} \cdot(16)
\end{gathered}
$$

Hierin ist nach (13)

$$
\left(\frac{\lambda}{k} K\right)^{2}=\left(\cos ^{2} \lambda+\frac{k^{2}}{\lambda^{2}} \sin ^{2} \lambda\right)^{-1} .
$$

Für die praktische Auswertung der Ergebnisse sind noch einige Vernachlässigungen zweckmäßig. Für $a \leqq 3 \cdot 10^{-13} \mathrm{~cm}$ wird $\gamma \leqq 0,761$ und nach (14) wird dann $x \leqq 3,24$ und $A / \varepsilon \geqq 17,0$. Andererseits ist $x>\pi$, und es kann nach der kleinen Größe $x-\pi$ entwickelt und mit dem ersten Glied abgebrochen werden. Dasselbe kann mit $\lambda-x$ gemacht werden, solange die eingestrahlte Energie $\hbar \omega$ nicht wesentlich größer als $2 \varepsilon$ wird, d.h. $k^{2}$ nicht wesentlich größer als $2 \gamma^{2}$. Die Bestimmungsgleichungen für $N_{1}, N_{2}$ vereinfachen sich dann sehr und liefern

$$
N_{1}^{2}=\frac{1}{2 \pi} \frac{(1+\gamma)^{2}}{1+\gamma+\gamma^{2 / 3}} .
$$

Im Ausdruck (16) kann im Faktor von $N_{2}$ die Größe $\lambda^{-1} \sin \lambda$ vernachlässigt werden. Nach einigen Umformungen wird, wenn noch näherungsweise

$$
2 \pi * \frac{\lambda-\psi}{\lambda}=\gamma^{2}+k^{2}
$$


gesetzt wird,

$$
\left|z_{0 e}^{0}\right|=\frac{4 \pi}{3} \frac{\left|N_{1}\right|}{\left(\gamma^{2}+k^{2}\right)^{2}}\left(\frac{2 \gamma^{2}}{1+\gamma}+\gamma^{2}+k^{2}\right)
$$

erhalten. Wird noch das Energieverhältnis $y=(\hbar \omega-\varepsilon) / \varepsilon=(k / \gamma)^{2}-$ d.h. der Überschuß über die Bindungsenergie $\varepsilon$ als Vielfaches von $\varepsilon-$ eingeführt, so ergibt sich schließlich

$$
\begin{array}{r}
\sigma_{\gamma^{\prime}}=\frac{16 \pi}{27}\left(\frac{Z M}{M_{R}}\right)^{2} \frac{e^{2}}{\hbar c} \frac{1 / \gamma}{1+\gamma+\gamma^{2 / 3}} \frac{\sqrt{y}}{(1+y)^{3}} \\
\cdot[2+(1+\gamma)(1+y)]^{2} .
\end{array}
$$

\section{2. $S-\mathrm{Zust}$ and}

Im folgenden soll die Rechnung für die Annahme durchgeführt werden, daß $u_{0}$ ein $S$-Zustand ist. Dann liefert von $\xi$ nur die Komponente $l=1$ einen Beitrag, und es wird

$$
z_{0 e}=\frac{4 \pi}{3} \cos \Theta \int_{0}^{\infty} u_{0} R_{1}^{*} r^{3} d r
$$

Dabei ist $\Theta$ der Winkel zwischen $\mathfrak{f}$ und der $z$-Richtung. Für genügend kleine Energien nimmt $R_{1}$ nur außerhalb der unmittelbaren Kernumgebung wesentliche Werte an, wo $V(r)$ praktisch verschwindet. In diesem Gebiet ist aber nach (1) angenähert

$$
u_{0}(r)=C r^{-1} \exp (-\gamma r)
$$

und

$$
\begin{gathered}
R_{1}(r)=\frac{3 i}{k r}\left(\frac{\sin k r}{k r}-\cos k r\right), \\
\frac{k}{3 i} \int_{0}^{\infty} u_{0} R_{1}^{*} r^{3} d r \\
=-\frac{C}{k} \operatorname{Re}\left[\int_{0}^{\infty}(k r+i) \exp (-\gamma+i k) r d r\right] \\
=-\frac{2 C k^{3}}{\left(\gamma^{2}+k^{2}\right)^{2}} .
\end{gathered}
$$

Bei der Mittelung über alle Raumrichtungen von $\mathfrak{f}$ wird $\overline{\cos ^{2} \Theta}=1 / 3$. Demnach wird jetzt

$$
\sigma_{\gamma}=\frac{16}{3} \pi^{2} \frac{e^{2}}{\hbar c}\left(\frac{Z M}{M_{R}}\right)^{2} \frac{C^{2}}{\gamma^{3}}\left(\frac{2 \sqrt{y}}{1+y}\right)^{3} .
$$

Hierbei ist nur noch die Größe $C$ vom Potentialverlauf $V(r)$ abhängig. a) Mit dem Ansatz (8) - jetzt mit einem anderen Wert $A$ - ergibt sich für den $S$-Zustand

$$
u_{0}(r)= \begin{cases}N \frac{\sin * r}{\psi r} & \text { für } r<1 \\ N \frac{\sin \%}{\varkappa r} \exp (\gamma-\gamma r) & \text { für } r>1,\end{cases}
$$

wobei sich jetzt $x$ und somit $A$ aus der Gleichung

$$
\frac{\operatorname{tg} x}{\%}=-1 / \gamma
$$

bestimmt. Die Normierungsbedingung für $u_{0}$ liefert jetzt

$$
\left(4 \pi N^{2}\right)^{-1}=\frac{1}{2}-\frac{\sin 2 \varkappa}{4 \%}+\frac{\sin ^{2} \varkappa}{\gamma} .
$$

Damit ist dann $C=N \exp (-\gamma) \sin \varkappa$ bestimmt.

$\beta)$ Es läßt sich hier auch vergleichsweise der Potentialansatz

$$
V(r)=-A \exp (-r)
$$

behandeln. Dafür wird

$$
r u_{0}(r)=N J_{q}[c \exp (-r / 2)]
$$

mit $q=2 \gamma, c^{2}=8 M A / \hbar^{2}$, wobei $c$ die erste Nullstelle der Bessel-Funktion $J_{q}$ sein muß; dadurch wird $A$ bestimmt. - Die Entwicklung für große $r$ und der Vergleich mit (19) ergibt jetzt $C=N(c / 2)^{q} / \Gamma(1+q)$. Die Größe $N$ bestimmt sich aus der Normierungsbedingung für $u_{0}$, die

$$
N^{2} 8 \pi \int_{0}^{c} J_{q}^{2}(t) \frac{d t}{t}=1
$$

geschrieben werden kann. Das dabei auftretende Integral kann berechnet werden, indem man die Besselsche Differentialgleichung für $J_{q}(t)$ zur daraus gebildeten Differentialgleichung für $\partial J_{q} / \partial t$ addiert und die so gewonnene Gleichung zwischen den Grenzen 0 und $c$ über $t$ integriert, wobei noch zu beachten ist, daß $\left(\partial J_{q} / \partial t\right)_{t=c}$ $=J_{q-1}(c)$. So wird die Gleichung

$$
\left(4 \pi N^{2}\right)^{-1}=-\frac{c}{q} J_{q-1}(c) \frac{\partial J_{q}(c)}{. \partial q}
$$

zur Bestimmung von $N$ erhalten.

Im Ausdruck (20) des Wirkungsquerschnittes für den $S$-Zustand ist nur ein energieunabhängiger Zahlenfaktor von der Annahme des Kernradius $a$ abhängig. Die numerische Auswertung zeigt, daß das in guter Näherung auch bei (17) für den $P$ Zustand gilt. Die Abhängigkeit des Wirkungs- 
querschnittes von der Einstrahlungsenergie ist in beiden Fällen ziemlich verschieden. Sie ist in der Abb. 1 dargestellt. Es wird in beiden Fällen ein Maximum durchlaufen, das für den $P$-Zustand bald nach Úberschreiten des Schwellenwertes bei 1,3-facher Bindungsenergie erreicht wird, für den $S$-Zustand aber erst bei doppelter Bindungsenergie. Der Unterschied ist dadurch begründet, daß im zweiten Fall ein Übergang zu einem $P$-Zustand des Kontinuums erfolgen muß, der aber bei kleinen Energien noch weit vom Kern entfernt ist.

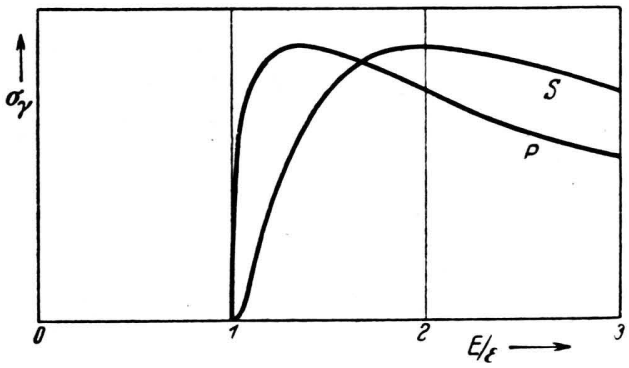

Abb. 1. Verlauf des Wirkungsquerschnittes beim Kernphotoeffekt in Abhängigkeit von der Einstrahlungsenergie; $P$ für einen $P$-Zustand, $S$ für einen $S-Z u-$ stand als Grundzustand. Die Maximalwerte sind willkürlich gleichgesetzt.

Es liegen Relativmessungen von $\sigma_{\gamma}$ für zwei verschiedene $\gamma$-Strahler von Fleischmann und Ge $n$ t $n$ e $r^{5}$ vor, mit denen die Ergebnisse für den $P$-Zustand wesentlich besser übereinstimmen als für den S-Zustand. Neuere Messungen der Neutronenausbeute für energiereiche Röntgenstrahlen von $\mathrm{Kop}$ ferman $\mathrm{n}$ und $\mathrm{Pa} \mathrm{u}^{6}{ }^{6}$ sowie von Wi ed e $\mathrm{nbeck}^{7}$ lassen noch keine Überprüfung der theoretischen Ergebnisse zu, solange nicht das Spektrum der Rüntgenstrahlung genauer bekannt ist. - Houtermans und Bartz geben den mittleren Wirkungsquerschnitt $\bar{\sigma}_{\gamma}$ für das $\gamma$-Spektrum des $\mathrm{RaB}+\mathrm{C}$. Sie geben gleichzeitig eine ausführliche Zusammenstellung älterer Messungen verschiedener Autoren, deren Werte zwischen $3 \cdot 10^{-28}$ und $20 \cdot 10^{-28} \mathrm{~cm}^{2}$ schwanken. Der von beiden Autoren selbst gemessene Wert ist

$$
\bar{\sigma}_{\gamma}=8,8 \pm 1,0 \cdot 10^{-28} \mathrm{~cm}^{2} .
$$

Das verwendete $\gamma$-Spektrum ist in der Arbeit zitiert. - In Tab. 1 sind die theoretischen Maximalwerte von $\sigma_{\gamma}$ und in Tab. 2 die theoretischen Mittel-

5 R. Fle ischmann u. W. Gentner, Z. Physik 100, 440 [1936].

${ }_{6} \mathrm{H}$. K o p f e r m a n n u. W. P a u l, Nachr. Ges. Wiss. Göttingen, Math.-physik. Kl., 1946, 17.

7 M. L. W i e d e n b e c k, Physic. Rev. 69, 235 [1946].

\begin{tabular}{|c|c|c|c|}
\hline \multirow{2}{*}{$\begin{array}{l}\text { Kernradius } a \\
\left(10^{-13} \mathrm{~cm}\right)\end{array}$} & \multicolumn{3}{|c|}{$\begin{array}{l}\text { Maximaler W.Q. }\left(\sigma_{\gamma}\right)_{\max } \\
\quad\left(10^{-28} \mathrm{~cm}^{2}\right)\end{array}$} \\
\hline & $P$-Zustand & $\begin{array}{l}S-Z \\
a)\end{array}$ & $\begin{array}{l}\text { nd } \\
\beta)\end{array}$ \\
\hline 1,5 & 3,59 & 26,8 & 133 \\
\hline 2,0 & 3,90 & 30,9 & 240 \\
\hline 2,5 & 4,23 & 37,3 & - \\
\hline 3,0 & 4,60 & 45,3 & - \\
\hline
\end{tabular}

'Tab. 1. Theoretische Maximalwerte des Wirkungsquerschnittes für verschiedene Werte des Kernradius a.

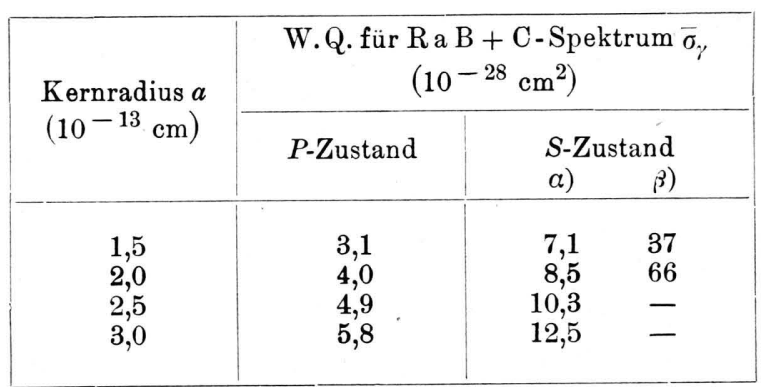

'Tab. 2. Theoretische Mittelwerte des Wirkungsquerschnittes für verschiedene Werte des Kernradius $a$.

werte $\bar{\sigma}_{\gamma}$ für verschiedene Werte $a$ des Kernradius angegeben. Dabei bezieht sich die erste Spalte $\alpha$ beim S-Zustand auf den Ansatz (8), die Spalte $\beta$ auf den Ansatz (22) der Kernkräfte. Die Größenordnung ist also für den Ansatz (8) sowohl beim $P$-Zustand wie beim $S$-Zustand dieselbe und in Übereinstimmung mit den Messungen. Nach dem Gesetz universeller Kerndichte ist $a=2,8 \cdot 10^{-13} \mathrm{~cm}$. Mit dem exponentiellen Kraftgesetz (22) ergeben sich dagegen zu große Wirkungsquerschnitte beim S-Zustand.

Zusammenfassend läßt sich sagen: Die gemessene Energieabhängigkeit des Wirkungsquerschnittes spricht für den $P$-Zustand. Die absolute Größe spricht für das Kraftgesetz kurzer Reichweite (8), das für beide Zustände die richtige Größenordnung gibt. Auch die zweite Tatsache ist plausibel, denn da der Rumpf schon aus mehreren Nukleonen besteht, wird das Exponentialgesetz (22) ein schlechteres Bild als der Ansatz (8) liefern, der einem Tröpfchenmodell entspräche.

\section{Die Neutronenstreuung}

Bei der Betrachtung des Kernphotoeffektes war es wesentlich, ob der Grundzustand als $P$ - oder

8 F. Houtermans u. I. B artz, Physik. Z. 44, 167 [1943]. 
als S-Zustand angenommen wird; denn bei diesem Prozeß gelten Auswahlregeln für die Drehimpulsquantenzahl. Es handelt sich dann in beiden Fällen um verschiedene Übergänge; d.h. der Prozeß verläuft dann grundsätzlich verschieden. Für die im folgenden betrachteten Prozesse gelten keine analogen Auswahlregeln, so daß dieser grundsätzliche Uñterschied wegfällt. Es soll deshalb zur Vereinfachung der Rechnung der Grundzustand, auch wenn er tatsächlich ein $P$-Zustand ist, näherungsweise als S-Zustand zum selben Energiewert behandelt werden. Das ist vor allem dadurch gerechtfertigt, daß nach dem statistischen Mittel über alle Orientierungen der Kerne im Raum gefragt wird. - Weiterhin handelt es sich bei den folgenden Prozessen um ein Dreikörpersystem - Kernrumpf und zwei Neutronen. Es kann aber der 8-mal schwerere Rumpf als im Schwerpunkt ruhend angenommen werden. Das bedeutet hier eigentlich nur, daß eine gewisse Richtungsabhängigkeit der Ausstrahlung vernachlässigt wird. Im Gegensatz hierzu war beim Kernphotoeffekt gerade die Bewegung des Kernrumpfes für den Prozeß verantwortlich.

Das gesamte betrachtete System besteht aus den beiden Teilen des Kernes - Rumpf und gebundenes Neutron - und einem freien Neutron, das mit dem Kern in Wechselwirkung tritt. Die Wechselwirkung zwischen den beiden Neutronen soll als Störung behandelt werden. Dann bewegen sich beim ungestörten System die beiden Neutronen unabhängig voneinander im Kraftfeld des feststehenden Kernrumpfes. Bei der Betrachtung des Streuvorganges beschränkt man sich auf solche Zustände des Systems, bei denen ein Neutron an den Rumpf gebunden bleibt. Im ungestörten System wird das freie Neutron nur am Kernrumpf gestreut; der Störung entspricht dann eine zusätzliche Streuung am gebundenen Neutron, wobei noch Austauscheffekte wirksam werden. Schon beim ungestörten System tritt also eine Streuwelle auf. Dadurch unterscheidet sich die vorliegende Rechnung von einer Bornschen Näherung, für die es wesentlich ist, daß im ungestörten System keine Streuung auftritt und die Zustände der freien Teilchen durch ebene Wellen beschrieben werden.

Für jedes der beiden Neutronen gilt im ungestörten System die Schröding e r - Gleichung (1), wobei jetzt $M$ die Neutronenmasse darstellt. Eines befindet sich im Zustand $u_{0}(r)$, das andere im Zu- stand $\xi$ (f, r $)$, der asymptotisch in die ebene Welle übergeht. In weiterer Näherung wird sein asymptotisches Verhalten durch

$$
\exp (i \mathfrak{f} x)+f(\alpha) \exp (i k r) / k r
$$

beschrieben. Der zweite Summand stellt dabei die Streuwelle dar, die vom Kernrumpf allein herrührt. - Die ungestörten Zustände sind diejenigen, die durch Symmetrisierung aus

$$
U_{0}(1,2)=V-1 / 2 u_{0}\left(r_{1}\right) \xi\left(\mathfrak{f}, \mathfrak{r}_{2}\right)
$$

entstehen :

$$
\begin{aligned}
& \Phi_{0}^{+}=2^{-1 / 2}\left[U_{0}(1,2)+U_{0}(2,1)\right], \\
& \Phi_{0}^{-}=2^{-1 / 2}\left[U_{0}(1,2)-U_{0}(2,1)\right] .
\end{aligned}
$$

Dabei ist $V$ ein grofses Normierungsvolumen und $\mathfrak{r}_{1}, \mathfrak{r}_{2}$ sind die Ortskoordinaten der beiden Neutronen. Es gehört nun der symmetrische Zustand $\Phi_{0}^{+}$zur antiparallelen Spinstellung der beiden Neutronen, der antisymmetrische Zustand $\Phi_{0}^{-}$zur parallelen Spinstellung. Die Wechselwirkung zwischen beiden Neutronen ist abhängig von der gegenseitigen Spinorientierung, die nur parallel oder antiparallel sein kann. Sie lasse sich durch zwei Potentialfunktionen des gemeinsamen $\mathrm{Ab}$ standes $r_{12}$ beschreiben: $W+\left(r_{12}\right)$ bzw. $W-\left(r_{12}\right)$ bei antiparalleler bzw. paralleler Spinstellung. Diese Energie tritt in der Behandlung als Störung auf. Die Zustände des gestörten Systems seien entsprechend den Symmetrieeigenschaften mit $\Phi^{+}$, $\phi^{-}$bezeichnet. Wenn $H_{i}$ der Energieoperator der Gl. (1) ist, der auf die Koordinate $r_{i}$ wirkt, dann gilt die Schrödinger-Gleichung:

$$
\left(H_{1}+H_{2}+W^{ \pm}-E+\varepsilon\right) \Phi^{ \pm}=0,
$$

wobei die oberen oder die unteren Zeichen zusammengehören. $E$ ist die Energie des eingestrahlten Neutrons. Wird $\Phi=\Phi_{0}+\Phi_{1}$ gesetzt, so gilt in erster Näherung

$$
\left(H_{1}+H_{2}-E+\imath\right) \omega_{\frac{ \pm}{1}}^{ \pm}=-W^{ \pm} \Phi_{\frac{1}{0}}^{ \pm} .
$$

Dabei muß $V$ genügend groß vorausgesetzt werden, so daß die Energiewerte praktisch kontinuierlich liegen.

Für die Korrektur $\Phi_{1}$ in erster Näherung sei ein Ansatz gewählt, der genau so wie $\Phi_{0}$ gebildet wird, indem nur $\xi(\mathfrak{r})$ durch eine andere Funktion $\varphi(\mathfrak{r})$ ersetzt wird. Diese Festsetzung bedeutet dann keine unzulässige Einschränkung, wenn. 
nach dem asymptotischen Verhalten der Lösung allein gefragt wird, wie das hier der Fall ist; denn asymptotisch läßt sich für große Abstände $r_{1}, r_{2}$ vom Kernzentrum auch die strenge Lösung in der gleichen Weise nach $\mathfrak{r}_{1}, \mathfrak{r}_{2}$ separieren.

Der Streuquerschnitt $\sigma_{n}$ ist das Verhältnis des Gesamtstromes der gestreuten Neutronen zur Stromdichte der einfallenden Neutronen. Wenn getrennt die Streuquerschnitte $\sigma_{n}^{+}$und $\sigma_{n}^{-}$für Neutronen antiparalleler bzw. paralleler Spinstellung zum gebundenen Neutron betrachtet werden, gilt entsprechend den statistischen Gewichten der beiden Spinzustände

$$
\sigma_{n}=\frac{1}{4} \sigma_{n}^{+}+\frac{3}{4} \sigma_{n}^{-} .
$$

Der fiktive Zustand, bei dem ein Neutron am Kern gebunden wäre und ein Neutron sich frei - ohne Wechselwirkung mit dem Kern - bewegen würde, soll $\Phi_{f}$ genannt werden. Er wird wie $\Phi_{0}$ gebildet, wenn an Stelle von $\xi$ die ebene Welle $\exp (i \mathfrak{f r})$ gesetzt wird. Dann entspricht $\Phi-\Phi_{f}$ der Streuwelle am gesamten Kern. Es ist dann

$$
\sigma_{n}^{ \pm}=2 \int_{F_{1}} d F_{1} \int d \mathfrak{r}_{2} \mid \Phi^{ \pm}-\Phi_{f}^{ \pm}{ }^{2},
$$

wenn über eine Fläche $F_{1}$ integriert wird, die den Kern umschließt und allseitig genügend weit von ihm entfernt ist. Wegen des exponentiellen Abfalles von $u_{0}\left(r_{1}\right)$ für große $r_{1}$ bleibt dann in der ersten Näherung asymptotisch nur

$$
\sigma_{n}^{ \pm}=\int_{F^{\prime}} d F \mid \xi(\mathfrak{x})+\varphi(\mathfrak{x})-\exp (i \mathfrak{f} \mathfrak{x}){ }^{2}
$$

übrig. Dabei ist $\varphi$ von der Spinstellung der Neutronen zueinander abhängig, was hier durch die Bezeichnung nicht besonders zum Ausdruck gebracht wird.

Die Aufgabe der Störungsrechnung ist die Berechnung von $\varphi$. Gl. (24) führt auf

$$
\begin{aligned}
\left(H_{2}-E\right) \varphi\left(\mathrm{r}_{2}\right)= & \mp u_{0}\left(r_{2}\right) \int d \mathfrak{r}_{1} u_{0}^{*}\left(r_{1}\right)\left(H_{1}-E\right) \varphi\left(\mathfrak{r}_{1}\right) \\
& -\xi\left(\mathfrak{r}_{2}\right) \int d \mathfrak{r}_{1} u_{0}^{*}\left(r_{1}\right) W^{ \pm} u_{0}\left(r_{2}\right) \\
& \mp u_{0}\left(r_{2}\right) \int d \mathfrak{r}_{1} u_{0}^{*}\left(r_{1}\right) W^{ \pm} \xi\left(\mathfrak{r}_{2}\right) .
\end{aligned}
$$

Das erste Integral ist eine Konstante, während die beiden anderen von $\mathfrak{r}_{2}$ abhängen. Die Integranden liefern nur für Werte $r_{1}$ in der Größenordnung der Kerndimensionen wesentliche Beiträge, weil jedesmal der Faktor $u_{0}^{*}\left(r_{1}\right)$ auftritt. Dann haben aber wegen der kurzen Reichweite der Wechselwirkung $W$ die beiden letzten Integrale nur für Abstände $r_{2}$ in Kerndimensionen wesentliche Werte und man kann sich bei genügend kleinen Energien auf die Komponente $l=0$ in der Entwicklung (6) von $\xi\left(\mathfrak{r}_{2}\right)$ beschränken. Die Integrale lassen sich dann über $r_{1}$ und den Winkel zwischen $\mathfrak{r}_{1}$ und $\mathfrak{r}_{2}$ erstrecken. Die ganze rechte Seite von (28) ist dann eine Funktion vom Betrag $r_{2}$ allein. Sie sei gemeinsam für beide Spinstellungen abgekürzt mit $-\left(\hbar^{2} / 2 M\right) \mu\left(r_{2}\right) / k r_{2}$ bezeichnet. Mit der Schreibweise $v(r)=V(r) 2 \mathrm{M} / \hbar$ und $\mathfrak{r}_{2}=\mathfrak{r}$ wird dann aus (28)

$$
\left[\Delta+k^{2}-v(r)\right] \varphi(\mathfrak{x})=\mu(r) / k r .
$$

Die Funktionen $\xi(\mathfrak{r})$ sind die Lösungen der zu (29) gehörigen homogenen Gleichung; sie sind im ungestörten Problem schon enthalten. Sie brauchen in $\varphi(x)$ nicht mehr einbezogen zu werden. In einer zu (6) analogen Entwicklung von $\varphi$ nach Kugelfunktionen von $\alpha$ wird bei den betrachteten Energien nur die Komponente $l=0$ wesentlich sein, da nur sie in den Bereich der Störung $\mu(r)$ reicht, so daß sich $\varphi(\mathrm{r})=s(r) / k r$ schreiben läßt und (29) zu einer gewöhnlichen Differentialgleichung wird:

$$
\frac{d^{2} s}{d r^{2}}+\left[k^{2}-v(r)\right] s=\mu(r) .
$$

Die homogene Gleichung dazu hat die Lösung $\chi$, die bei $r=0$ verschwindet und für große $r$ asymptotisch in $\sin (k r+\delta)$ übergeht. Die zweite unabhängige Lösung $\eta$ soll asymptotisch in $\cos (k r+\delta)$ übergehen. Die Lösung $s(r)$ muß für $r=0$ auch verschwinden und kann deshalb nur die Gestalt

$$
\begin{aligned}
s(r)=D \%(r) & +\psi(r) \int_{0}^{r} \mu\left(r^{\prime}\right) \eta\left(r^{\prime}\right) d r^{\prime} \\
& -\eta(r) \int_{0}^{r} \mu\left(r^{\prime}\right) \%\left(r^{\prime}\right) d r^{\prime}
\end{aligned}
$$

haben, mit einer Konstanten $D$. Die gesamte Streuwelle in (27) ist asymptotisch

$\xi+\varphi-\exp (i \mathfrak{f} \mathfrak{x})=\rightarrow[\chi(r)-s(r)-\sin k r] / k r$.

Da die Integranden in (31) nur für genügend kleine $r^{\prime}$ Beiträge liefern, können die oberen Grenzen der Integrale im asymptotischen Ausdruck für s $(r)$ durch $\infty$ ersetzt werden. Dann läßt sich mit den Abkürzungen 


$$
\beta=\int_{0}^{\infty} u \chi d r, \quad \beta^{\prime}=1+D+\int_{0}^{\infty} \mu \eta d r
$$

schreiben:

$$
\begin{aligned}
\xi+ & \varphi-\exp (i \mathfrak{f r}) \\
& =\rightarrow \frac{\exp (i(k r+\delta))}{2 i k r}\left[\beta^{\prime}-i \beta-\exp (-i \delta)\right] \\
- & \frac{\exp (-i(k r+\delta))}{2 i k r}\left[\beta^{\prime}+i \beta-\exp (i \delta)\right] .
\end{aligned}
$$

Die Konstante $D$ ergibt sich durch die Ausstrahlungsbedingung, nach der der Faktor von $\exp (-i k r)$, der einer einfallenden Welle entspräche, verschwinden muß. Es wird dann die rechte Seite von (33) gleich $(\sin \delta-\beta)$ $\cdot \exp (i[k r+\delta]) / k r$. Wenn nun wieder zwischen den beiden Spineinstellungen der Neutronen unterschieden wird, ergibt sich schließlich

$$
\sigma_{n}^{ \pm}=\frac{4 \pi}{k^{2}}\left|\sin \delta-\beta^{ \pm}\right|^{2} .
$$

$\beta$ wird nach (32) gebildet, indem für $\mu$ der ursprüngliche Ausdruck - d.h. bis auf Faktoren die rechte Seite von (28) - eingesetzt wird. Wegen der Orthogonalität von $u_{0}$ und $\xi$ fällt der Anteil weg, der vom ersten Summanden dieses Ausdrukkes herrührt. Es ergibt sich

$$
\beta^{ \pm}=\frac{k}{4 \pi} \frac{2 M}{\hbar^{2}}\left(W_{00}^{ \pm} \pm A_{00}^{ \pm}\right),
$$

wenn die Bezeichnungen

$$
\begin{aligned}
W_{00}^{ \pm} & =V \int d \mathfrak{r}_{1} d \mathfrak{r}_{2} U_{0}^{*}(1,2) W^{ \pm} U_{0}(1,2), \\
A_{00}^{ \pm} & =V \int d \mathfrak{r}_{1} d \mathfrak{r}_{2} U_{0}^{*}(1,2) W^{ \pm} U_{0}(2,1)
\end{aligned}
$$

gewählt werden. Mit der Abkürzung $x=\cos \left(\mathfrak{r}_{1}, \mathfrak{r}_{2}\right)$ ist

$$
\begin{gathered}
W_{00}^{ \pm}=\frac{8 \pi^{2}}{k^{2}} e^{i \delta} \int_{0}^{\infty} d r_{1} \int_{0}^{\infty} d r_{2} \int_{-1}^{+1} d x r_{1} u_{0}^{*}\left(r_{1}\right) \chi^{*}\left(k, r_{2}\right) \\
\cdot W^{ \pm}\left(r_{12}\right) r_{1} u_{0}\left(r_{1}\right) \chi\left(k, r_{2}\right), \\
A_{\eta_{0}}^{ \pm}=\frac{8 \pi^{2}}{k^{2}} e^{i \delta} \int_{0}^{\infty} d r_{1} \int_{0}^{\infty} d r_{2} \int_{-1}^{+1} d x r_{1} u_{0}^{*}\left(r_{1}\right) \chi^{*}\left(k, r_{2}\right) \\
\cdot W^{ \pm}\left(r_{12}\right) \chi\left(k, r_{1}\right) r_{2} u_{0}\left(r_{2}\right) .
\end{gathered}
$$

Für eine Neutron-Neutron-Wechselwirkung

$$
W^{ \pm}\left(r_{12}\right)=-B^{ \pm} \exp \left(-r_{12}^{2} / b^{2}\right)
$$

lassen sich die Integrale in (37) leicht separieren. Dann wird

$$
\begin{aligned}
& \int_{-1}^{+1} W^{ \pm}\left(r_{12}\right) d x \\
& =-B^{ \pm} \exp \left(-\frac{r_{1}^{2}+r_{2}^{2}}{b^{2}}\right) \frac{b^{2}}{r_{1} r_{2}} \sin \mathrm{h} \frac{2 r_{1} r_{2}}{b} \\
& =-2 B^{ \pm} \exp \left(-\frac{r_{1}^{2}+r_{2}^{2}}{b^{2}}\right) \sum_{n=0}^{\infty} \frac{2^{2 n}}{(2 n+1) !\left(\frac{r_{1} r_{2}}{b^{2}}\right)^{2 n},}
\end{aligned}
$$

und es läßt sich schreiben:

$$
\begin{aligned}
& \int W_{00}^{ \pm}=-\frac{4 \pi^{3}}{k^{2}} e^{i} \delta^{2} B^{ \pm} \sum_{n=0}^{\infty} \frac{2^{2 n}}{(2 n+1) !} G_{n} ， \\
& A_{00}^{ \pm}=-\frac{4 \pi^{3}}{k^{2}} e^{i \delta} b^{2} B^{ \pm} \sum_{n=0}^{\infty} \frac{2^{2 n}}{(2 n+1) !} \widetilde{G}_{n}, \\
& \left\{\begin{aligned}
G_{n}= & \frac{4}{\pi} \int_{0}^{\infty} \exp \left(-r^{2} / b^{2}\right)(r / b)^{2 n} r^{2}\left|u_{0}(r)\right|^{2} d r \cdot b \\
& \cdot \int_{0}^{\infty} \exp \left(-r^{2} / b^{2}\right)(r / b)^{2 n}|\chi(k, r)|^{2} d r / b,
\end{aligned}\right. \\
& \widetilde{G}_{n}=\frac{4}{\pi}\left|\int_{0}^{\infty} \exp \left(-r^{2} / b^{2}\right)(r / b)^{2 n} r u_{0}^{*}(r) \chi(k, r) d r / b\right|^{2} .
\end{aligned}
$$

Mit dem Ansatz (8) für das Potential $V(r)$ lassen sich die Integrale in (40) relativ einfach auswerten. Man wird/den Neutronenradius $b$ kleiner als den Radius 1 des Kernrumpfes annehmen. Dann kann man für die Lösungen $\chi, u_{0}$ nach (9), (21) die Ausdrücke für $r<1$ einsetzen, weil die Integranden - zumindest für kleine $n-$ dann für $r>1$ keine wesentlichen Beiträge mehr liefern. Es seien zur Berechnung der Integrale dann die Hilfsfunktionen

$$
\varphi_{n}(p)=\frac{2}{\sqrt{\pi}} \int_{0}^{\infty} \exp \left(-t^{2}\right) t^{2 n} \cos p t d t
$$

für ganzzahliges $n$ eingeführt, die sich auf Grund der Relationen

$$
\varphi_{n}=(-1)^{n} \frac{d^{2 n} \varphi_{0}}{d p^{2 n}}, \quad \varphi_{0}=\exp \left(-p^{2} / 4\right)
$$

auf die Hermiteschen Polynome

$$
H_{n}(x)=(-1)^{n} \exp \left(x^{2} / 2\right) \frac{d^{n}}{d x^{n}} \exp \left(-x^{2} / 2\right)
$$

zurückführen und numerisch berechnen lassen:

$$
\varphi_{n}(p)=(-1 / 2)_{.}^{n} \exp \left(-p^{2} / 4\right) H_{2 n}(p / \sqrt{2)} .
$$


Mit

$$
\begin{aligned}
f_{n}\left(p_{1}, p_{2}\right) & =\frac{2}{\sqrt{\pi}} \int_{0}^{\infty} \exp \left(-t^{2}\right) t^{2 n} \sin p_{1} t \sin p_{2} t d t \\
& =\frac{1}{2}\left[\varphi_{n}\left(p_{1}-p_{2}\right)-\varphi_{n}\left(p_{1}+p_{2}\right)\right]
\end{aligned}
$$

wird dann

$$
\begin{aligned}
& G_{n}=N^{2} K^{2} f_{n}(\varkappa b, \varkappa b) f_{n}(\lambda b, \lambda b), \\
& \widetilde{G}_{n}=N^{2} K^{2} f_{n}^{2}(\varkappa b, \lambda b) .
\end{aligned}
$$

Die Auswertung zeigt, daß die ersten Glieder der Reihen in (39) mit wachsenden $n$ rasch klein gegenüber denen für $n=0$ werden. Für größere $n$ konvergieren sie langsamer; aber gerade dafür wird die Näherung ungültig, die mit den Ausdrücken von $\chi, u_{0}$ für $r<1$ rechnet. Die Näherungsausdrücke für $G_{n}, \widetilde{G}_{n}$ werden für größere $n$ zu groß. Das gilt aber für Glieder der Reihen, die selbst schon klein gegenüber dem nullten Glied sind. Das einzelne Glied hat dann selbst schon keinen wesentlichen Einfluß mehr, aber die vollständige Entwicklung würde nach der Näherung zu große Werte liefern. Dem wird dadurch Rechnung getragen, daß die Reihen dort abgebrochen werden, wo das einzelne Glied klein ist und $r=b n^{1 / 2}$, die Maximalstelle von $\exp \left(-r^{2} / b^{2}\right)$ $\cdot(r / b)^{2 n}$, größer als 1 ist. - Weiter zeigt die numerische Auswertung, daß nach (35)

$$
\beta^{ \pm}=-\left|\beta^{ \pm}\right| e^{i \delta}
$$

ist. o ergibt sich aus (13). Es wird nach (34)

wobei

$$
\sigma_{n}^{ \pm}=\sigma_{n}^{\prime}+\sigma_{n}^{\prime \prime \pm}+2\left(\sigma_{n}^{\prime} \sigma_{n}^{\prime \prime \pm}\right)^{1 / 2} \cos \delta
$$

$$
\sigma_{n}^{\prime}=\frac{4 \pi}{k^{2}} \sin ^{2} \delta
$$

der Streuquerschnitt des Kernrumpfes allein ist und

$$
\sigma_{n}^{\prime} \pm=\frac{4 \pi}{k^{2}}\left|\beta^{ \pm}\right|^{2}
$$

je nach der Spinstellung der Streuquerschnitt ist, der dem gebundenen Neutron allein zukäme, wenn der Rumpf nicht streuen würde. Der dritte Summand in (44) entspricht der Interferenz zwischen

9 G. B r e it, E. U. C ond on u. R. D. Present, Physic. Rev. 50, 886, 825 [1936]; s. a. S. F l ü g g e u. A. K r e b s, Physik. Z. 38, 30 [1937].

10 H. D o l c h, Z. Physik 100, 401 [1936].

11 M. G o l d h a b e r u. G. H. B r i g g s, Proc. Roy. Soc. [London], Ser. A 162, 127 [1937].

\begin{tabular}{|c|c|}
\hline $\begin{array}{c}\text { Einstrahlungs- } \\
\text { energie } E / \varepsilon\end{array}$ & $\begin{array}{c}\text { Streuquerschnitt } \sigma_{n} \\
\left(10^{-24} \mathrm{~cm}^{2}\right)\end{array}$ \\
\hline 0,0 & 5,25 \\
0,4 & 3,04 \\
0,8 & 1,98 \\
1,2 & 1,43 \\
1,6 & 1,09 \\
\hline
\end{tabular}

Tab. 3. Streuquerschnitte $\sigma_{n}$ für $a=3 \cdot 10^{-13} \mathrm{~cm}$.

den beiden Streuzentren - Rumpf und Neutron. Der gesamte Streuquerschnitt des ganzen Kernes gegenüber Neutronen aller Spinrichtungen setzt sich nach (25) zusammen.

Die Werte $B^{+}$für die Neutron-Neutron-Wechselwirkung sind der Proton-Proton-Streuung, die Werte $B^{-}$der Deuteron-Bindungsenergie entnommen; und zwar ist nach Breit, Cond on und Presen ${ }^{9}$

$$
\begin{array}{ll}
B^{+}=6,16 m c^{2} & \text { für } b=1,74 \cdot 10^{-13} \mathrm{~cm}, \\
B^{+}=39 m c^{2} & \text { für } b=2,17 \cdot 10^{-13} \mathrm{~cm},
\end{array}
$$

nach D o l c h ${ }^{10}$

$$
B^{-}=122 m c^{2} \quad \text { für } b=1,74 \cdot 10^{-13} \mathrm{~cm} .
$$

Die Ergebnisse für den Streuquerschnitt $\sigma_{n}$ zeigen sich in weitem Bereich praktisch unabhängig von den Radien $a$ und $b$. Tab. 3 zeigt die Werte für $a=3 \cdot 10^{-13} \mathrm{~cm}$ zu verschiedenen Einstrahlungsenergien $E$. Für thermische Neutronen geben Goldhaber und Brigg sil den empirischen Streuquerschnitt $6,9 \cdot 10^{-24} \mathrm{~cm}^{2}$ an. Dunning ${ }^{12}$ u. a. finden nach älteren Messungen als totalen Wirkungsquerschnitt für thermische Neutronen $5,3 \cdot 10^{-24} \mathrm{~cm}^{2}$ und für Neutronen des Ra $\alpha+B e$ $1,63 \cdot 10^{-24} \mathrm{~cm}^{2}$. Demnach wird die Größenordnung und der starke Abfall mit wachsender Energie durch die Rechnung wiedergegeben. Nach A maldi u. a. ${ }^{13}$ beträgt für $0,37 \mathrm{MeV}$ der Streuquerschnitt $2,6 \cdot 10^{-24} \mathrm{~cm}^{2}$, nach Leipunski u. a. ${ }^{14}$ für $0,2 \mathrm{MeV} 2,9 \cdot 10^{-24} \mathrm{~cm}^{2}$. Le i p unsk i ${ }^{15}$ gibt für $0,9 \mathrm{MeV}$ den totalen Wirkungsquerschnitt $1,7 \cdot 10^{-24} \mathrm{~cm}^{2}$ an, Good und Scharff - Gold $\mathrm{h}$ a b e $\mathrm{r}^{16}$ nach neueren Messungen $2,8 \cdot 10^{-24} \mathrm{~cm}^{2}$. Der theoretische Wert ist $2,6 \cdot 10^{-24} \mathrm{~cm}^{2}$. - Neuere

12 I. R. D unning u. a., Physic. Rev. 48, 265 [1935].

13 E. A m a l d i u. a., Physic. Rev. 56, 881 [1939].

${ }_{14}$ L e i p unski u.a., Physik. Z. Sowjetunion 10, 625 [1936].

${ }_{15}$ L e i p unski, J. exp. theoret. Physik [MoskauLeningrad] 3, 231 [1940].

16 Good u. Scharff-Goldhaber, Physic. Rev. 59, 917 [1941]. 
Messungen von Allen, Burcham und Wil$\mathrm{kins}$ o $\mathrm{n}^{17}$ zeigen weitere Details in der Energieabhängigkeit, die allerdings nicht mehr von der Theorie des Zweikörpermodells wiedergegeben werden können. Es zeigt sich eine Resonanzstelle bei etwa doppelter Bindungsenergie.

\section{Der $(n, 2 n)$-Prozeß}

Im folgenden wird der Prozeß betrachtet, daß unter Einwirkung von Neutronenstrahlung ein Neutron aus dem Berylliumkern abgetrennt wird. Für das bei der Neutronenstreuung behandelte Modell ist der Prozeß ein Übergang des gesamten Dreikörpersystems von einem Zustand, bei dem ein Neutron an den Rumpf gebunden ist, zu einem Zustand, bei dem beide Neutronen frei sind. Im Rahmen einer Störungsrechnung, die wieder die Wechselwirkung zwischen den Neutronen als Störung behandelt, ist er ein Übergang des ungestörten Systems vom Anfangszustand $\Phi_{0}^{+}$oder $\Phi_{0}^{-} \mathrm{zu}$ einem Endzustand

mit

$$
\Phi_{e}^{ \pm}=2^{-1 / 2}\left[U_{e}(1,2) \pm U_{e}(2,1)\right]
$$

$$
U_{e}(1,2)=V^{-1} \xi\left(\mathfrak{f}_{1}, \mathfrak{r}_{1}\right) \xi\left(\mathfrak{f}_{2}, \mathfrak{r}_{2}\right) .
$$

Nach dem Diracschen Störungsverfahren ergibt sich die Wahrscheinlichkeit, daß das System während der Zeit $t$ vom Zustand $\Phi_{0}^{+}$mit der Energie $E_{0}$ unter Wirkung der Störung $W^{+}$in den $\mathrm{Zu}^{-}$ stand $\Phi_{e}^{+}$mit der Energie $E_{e}$ übergeht, als

$$
\left.a_{e}\right|^{2}=4 V^{-3}\left|M_{0 e}^{+}\right|^{2}\left(E_{0}-E_{e}\right)^{-2} \sin ^{2}\left(E_{0}-E_{e}\right) t / 2 \hbar,
$$

wenn

$$
M_{0 e}^{+}=V^{3 / 2} \int d \mathfrak{r}_{1} d \mathfrak{r}_{2}\left(\Phi_{e}^{+}\right)^{*} W^{+} \Phi_{0}^{+} .
$$

Bei diesem Übergang bleiben die Spins beider Neutronen antiparallel. Die Gleichungen gelten für parallele Spinstellung genau so, wenn das Pluszeichen als oberer Index durch das Minuszeichen ersetzt wird. Es ist zweckmäßig, das Matrixelement

$$
M_{\overline{0} e}^{+}=W_{\overline{0} e}^{+} \pm A_{\overline{0} e}^{\frac{t}{\partial}}
$$

zu schreiben, wobei

$$
\begin{aligned}
& W_{\overline{0} e}^{ \pm}=\int d \mathfrak{r}_{1} d \mathfrak{r}_{2} \xi^{*}\left(\mathfrak{f}_{1}, \mathfrak{r}_{1}\right) \xi^{*}\left(\mathfrak{f}_{2}, \mathfrak{r}_{2}\right) W^{ \pm} u_{0}\left(r_{1}\right) \xi\left(\mathfrak{f}, \mathfrak{r}_{2}\right), \\
& A_{\overline{0} e}^{ \pm}=\int d \mathfrak{r}_{1} d \mathfrak{r}_{2} \xi^{*}\left(\mathfrak{f}_{1}, \mathfrak{r}_{1}\right) \xi^{*}\left(\mathfrak{f}_{2}, \mathfrak{r}_{2}\right) W^{ \pm} \xi\left(\mathfrak{f}, \mathfrak{r}_{1}\right) u_{0}\left(r_{2}\right) .
\end{aligned}
$$

17 K. W. Allen, W. E. B u reh a m u. D. H. Wi l kin s on, Nature [London] 159, 474 [1947].
Es geht $A_{0 e}$ durch Vertauschen von $\mathfrak{f}_{1}, \mathfrak{f}_{2}$ aus $W_{0 e}$ hervor. (45) gibt die Wahrscheinlichkeit des Übergangs während der Zeit $t$ in einen Endzustand mit ganz bestimmten Werten $\mathfrak{f}_{1}, \mathfrak{f}_{2}$. Die Wahrscheinlichkeit des Übergangs pro Zeiteinheit in irgendeinen der Endzustände mit beliebigen Werten $\mathfrak{f}_{1}, \mathfrak{f}_{2}$ ist

$$
w=\frac{1}{t} \sum_{e}\left|a_{e}\right|^{2},
$$

wobei über alle möglichen Endzustände $\Phi_{e}^{+}$gleichen Gesamtspins zu summieren ist. Der Operator $W$ wirkt nicht auf die Spinvariablen, so daß sich der Spinzustand nicht ändert. Es ist also nur über die Eigenwerte $\mathfrak{f}_{1}, \mathfrak{f}_{2}$ zu summieren. Bei genügend großem Normierungsvolumen $V$, in dem nur in einem verschwindenden Bruchteil die Funktion $\xi$ von einer kräftefreien Welle verschieden ist, läßt sich die Statistik kräftefreier Wellen anwenden, nach der die Anzahl der Eigenwertpaare $\mathfrak{l}_{1}, \mathfrak{f}_{2}$ mit Beträgen in den Intervallen $d k_{1}, d k_{2}$ und Richtungen in den Raumwinkeln $d \Omega_{1}, d \Omega_{2}$ gleich $V^{2}(2 \pi)^{-6} k_{1}^{2} k_{2}^{2} d k_{1} d k_{2} d \Omega_{1} d \Omega_{2}$ wird. So kann die Summation in (48) in eine Integration verwandelt werden. Die Wahrscheinlichkeit $w$, daß in der Zeiteinheit der Prozeß $(n, 2 n)$ erfolgt, wird der Wahrscheinlichkeit gleichgesetzt, daß das eingestrahlte Neutron bei Abwesenheit des Kernes den Wirkungsquerschnitt $\sigma_{2 n}^{+}$durchschritten hat. $k \hbar / M$ ist die ursprüngliche Neutronengeschwindigkeit; also $\sigma_{2 n}^{+}=V w M / k \hbar$. Wird statt der Variablen $k_{2}$ die Variable $z=\left(E_{0}-E_{e}\right) t / 2 \hbar$ eingesetzt, so ergibt sich

$$
\sigma_{2 n}^{+}=\frac{2}{(2 \pi)^{6}} \frac{M^{2}}{k \hbar^{4}} \int d \Omega_{1} d \Omega_{2} d k_{1} d z k_{1}^{2} k_{2} \mid \boldsymbol{M}_{0 e}^{+} e^{2} \frac{\sin ^{2} z}{z^{2}} .
$$

Dabei liefert nach genügend langer Zeit der Integrand nur an der Resonanzstelle $x=0$, d. h. für Erfüllung des Energiesatzes $k_{2}^{2}=k^{2}-k_{1}^{2}-\gamma^{2}$, einen Beitrag, wobei $\gamma$ nach (5) definiert ist. Die Energien sollen als Vielfache der Bindungsenergie $\varepsilon$ folgendermaßen bezeichnet werden:

$$
x_{1}=E_{1} / \varepsilon=k_{1}^{2} / \gamma^{2}, \quad y=(E-\varepsilon) / \varepsilon=k^{2} / \gamma^{2}-1,
$$

d. h. die Energie zur Wellenzahl $\mathfrak{f}_{1}$ mit $x_{1}$, der Überschuß der eingestrahlten Energie über die Bindungsenergie mit $y$. Dann ergibt sich

$$
\sigma_{2 n}^{+}=\frac{1}{2(2 \pi)^{3}} \frac{\gamma^{7}}{\varepsilon^{2}}(1+y)^{-1 / 2} \int_{0}^{y} d x_{1} \sqrt{x_{1}\left(y-x_{1}\right)} \overline{\mid M_{0 e}^{+{ }^{2}}} .
$$


Dabei soll der Querstrich über dem Absolutquadrat des Matrixelementes die Mittelung über alle Raumrichtungen von $\mathfrak{f}_{1}, \mathfrak{f}_{2}$ bedeuten. Der Wurzelausdruck unter dem Integral stellt als Funktion von $x_{1}$ einen Halbkreis um den Punkt $x_{1}=y / 2$ mit dem Radius $y / 2$ dar. Für kleine Energien $y$ wird sich der übrige Anteil des Integranden nur wenig mit $x_{1}$ ändern und kann für $x_{1}=y / 2$, d. h. für

$$
k_{1}^{2}=k_{2}^{2}=\left(k^{2}-\gamma^{2}\right) / 2=\bar{k}^{2},
$$

vor das Integral gezogen werden. Diese zweite Mittelwertbildung über alle $x_{1}$ soll durch einen zweiten Querstrich angedeutet werden:

$$
\sigma_{2 n}^{+}=2^{-7} \boldsymbol{\tau}^{-2} y^{2}(1+y)^{-1 / 2} \gamma^{7} \varepsilon^{-2} \overline{\left|M_{0 \boldsymbol{e}}^{+}\right|^{2}} .
$$

In welchem Bereich von $y$ das gültig ist, soll später untersucht werden. - Bei genügend kleinen Energien kann man sich wegen der geringen Reichweite von $u_{0}\left(r_{1}\right)$ und $W\left(r_{12}\right)$ in den Integralen (47) auf das Glied $l=0$ der Entwicklung (6) von $\xi$ beschränken. Dann hängen diese Integrale und somit $M_{0 e}$ nicht mehr von den Ausstrahlrichtungen $\mathfrak{f}_{1}, \mathfrak{f}_{2}$ ab; und die Mittelwertbildung über alle Richtungen fällt weg. Für (51) węrden dann die Integrale (47) einander gleich, und es wird

$$
\overline{\overline{\left|M_{0 e}^{+}\right|^{2}}}=4 \overline{\left.W_{0 e}^{+}\right|^{2}}, \quad \overline{\overline{M_{0 e}^{-\left.\right|^{2}}}}=0 .
$$

Der Querstrich über $W_{0 e}$ deutet dabei die Erfüllung von (51) an. Für den Wirkungsquerschnitt $\sigma_{2 n}^{-}$für Neutronen parallelen Spins zum Kern gilt (52) analog. Er verschwindet also nach (53) in der Näherung für kleine $y$. Der gesamte Wirkungsquerschnitt setzt sich entspr. (25) aus den Anteilen für verschiedene Spinorientierung zusammen. Das heißt $\sigma_{2 n}=\sigma_{2 n}^{+} / 4$. Der gesamte Wirkungsquerschnitt

$$
\sigma_{2 n}=2^{-7} \pi^{-2} y^{2}(1+y)^{-1 / 2} \gamma^{7} \varepsilon^{-2} \overline{\left|W_{0 e}^{+}\right|^{2}}
$$

rührf́ also nur von Neutronen antiparalleler Spinstellung her, da nach dem Pauli-Prinzip Neutronen parallelen Spins bei den betrachteten Energien einander nicht genügend nahe kommen, um miteinander $\mathrm{zu}$ reagieren.

Mit den Abkürzungen $\varrho=\delta(k)-\delta\left(k_{1}\right)-\delta\left(k_{2}\right)$ und $x=\cos \left(\mathrm{r}_{1}, \mathrm{r}_{2}\right)$ wird

$$
\begin{array}{r}
W_{0 e}^{+}=\frac{8 \pi^{2} e^{i} Q}{k k_{1} k_{2}} \int_{0}^{\infty} d r_{1} \int_{0}^{\infty} d r_{2} \int_{-1}^{+1} d x z^{*}\left(k_{1}, r_{1}\right) \psi^{*}\left(k_{2}, r_{2}\right) \\
\cdot W^{+}\left(r_{12}\right) r_{1} u_{0}\left(r_{1}\right) \chi\left(k, r_{2}\right) .
\end{array}
$$

Für die Neutron-Neutron-Wechselwirkung (38) wird

$$
W_{0 e}^{+}=-\frac{4 \pi^{3} b^{2} B^{+} e^{i} \varrho}{k k_{1} k_{2}} \sum_{n=0}^{\infty} \frac{2^{2 n}}{(2 n+1) !} G_{n},
$$

wobei jetzt

$$
\begin{aligned}
G_{n}= & \frac{4}{\pi} \int_{0}^{\infty} \exp \left(-r^{2} / b^{2}\right)(r / b)^{2 n} r u_{0}(r) \chi^{*}\left(k_{1}, r\right) d r / b \\
& \cdot \int_{0}^{\infty} \exp \left(-r^{2} / b^{2}\right)(r / b)^{2 n} \psi^{*}\left(k_{2}, r\right) \chi(k, r) d r / b .
\end{aligned}
$$

Diese Integrale lassen sich für den Potentialansatz (8) in derselben. Weise wie die analogen (40) beim Streuprozeß näherungsweise auswerten. Mit den dort definierten Funktionen (42) wird

$$
G_{n}=N K K_{1} K_{2} f_{n}\left(\varkappa b, \lambda_{1} b\right) f_{n}\left(\lambda b, \lambda_{2} b\right) .
$$

Die Indizes an $K$ und $\lambda$ entsprechen den Indizes von $k$. Für die Konvergenz der Reihenglieder in (56) gilt dasselbe wie bei den analogen Reihen in (40) bei der Neutronenstreuung.

Die Güte der Näherung, mit der das Integral in (50) ausgewertet wurde, kann nachträglich geprüft werden, indem man für einen festen Wert $y$ das Matrixelement $W_{0 e}^{+}$für verschiedene Werte $x_{1}$ berechnet und den Verlauf des Integranden in (50) mit dem Näherungsausdruck - in geeignetem Maßstab ein Halbkreis - vergleicht. Ist die Abweichung voneinander vernachlässigbar, dann ist die Näherung auch sicher für alle kleineren Werte $y$ zulässig. Eine Prüfung auf diese Art zeigt, daß die Näherung noch für $y=2$ sehr gut ist, wo schon die Beiträge $l>0$ wirksam zu werden beginnen.

Bei dieser Rechnung wird vorausgesetzt, daß der Neutronenradius $b$ kleiner als der Kernrumpfradius ist. Aus einem Grund, der weiter unten angegeben wird, wurde auch der Grenzfall betrachtet, daß $b$ groß gegenüber dem Kernrumpfradius 1 sei; dann ist $r u_{0}=(\gamma / 2 \pi)^{1 / 2} e^{-\gamma r}, \chi=\sin k r$ und

$$
G_{n}=(\gamma / 2 \pi) 1 / 2 S_{2 n}\left(\gamma b, k_{1} b\right) f_{n}\left(k b, k_{2} b\right)
$$

mit der Bezeichnung

$$
S_{n}(x, y)=\frac{2}{\sqrt{\pi}} \int_{0}^{\infty} \exp \left(-t^{2}-x t\right) t^{n} \sin y t d t .
$$

Diese Funktionen lassen sich durch Potenzreihenentwicklung des Sinus auch 


$$
S_{n}(x, y)=\sum_{k=0}^{\infty}(-1)^{k} \frac{y^{2 k+1}}{(2 k+1) !} F_{n+2 k+1}(x)
$$

mit

$$
F_{n}(x)=\frac{2}{\sqrt{\pi}} \int_{0}^{\infty} \exp \left(-t^{2}-x t\right) t^{n} d t
$$

schreiben. Aus der Gleichung $d g_{n} / d t=n g_{n-1}$ $-2 g_{n+1}$, die für $g_{n}(t)=\exp \left(-t^{2}\right) t^{n}$ gilt, folgen die Rekursionsformeln

$$
\begin{gathered}
F_{n+1}(x)=\frac{n}{2} F_{n-1}(x)-\frac{x}{2} F_{n}(x) \quad \text { für } n>1, \\
F_{1}(x)=\frac{1}{\sqrt{\pi}}-\frac{x}{2} F_{0}(x),
\end{gathered}
$$

durch die man die Berechnung von $F_{n}$ und $S_{n}$ auf

$$
F_{0}(x)=\frac{2}{\sqrt{\pi}} \exp \left(x^{2} / 4\right) \int_{x / 2}^{\infty} \exp \left(-t^{2}\right) d t
$$

zurückführen kann. Dieser Ausdruck setzt sich aus tabellierten Funktionen zusammen.

Die berechneten Werte für den Wirkungsquerschnitt $\sigma_{2 n}$ ergeben sich in weitem Bereich als praktisch unabhängig von der Annahme des Neutronenradius $b$, hängen aber stark vom Kernrumpfradius $a$ ab. In Tab. 4 sind die theoretischen Werte $\sigma_{2 n}$ für die beiden Werte $a=2 \cdot 10^{-13} \mathrm{~cm}$ (Spalte I) und $a=3 \cdot 10^{-13} \mathrm{~cm}$ (Spalte II) in Abhängigkeit von der Einstrahlungsenergie $E$ angegeben. Da es sich um größenordnungsmäßige Betrachtungen handelt, wurde die Rechnung bis $y=2$ ausgedehnt, wo zwar die Komponente $l=1$ in der Entwicklung (6) schon einen gewissen Beitrag liefert, aber sicher noch den von $l=0$ nicht überwiegt. In Tab. 5 sind die Werte $\sigma_{2 n}$ für $E / \varepsilon=3$ in Abhängigkeit von $a$ angegeben. Sie wachsen mit abnehmendem $a$ stark an, weil bei kleinerem verfügbaren Raum für beide Neutronen die Wahrscheinlichkeit, miteinander zu reagieren, größer wird. Um zu sehen, ob bei genügend kleinem a dieser Anstieg Größenordnungen ausmachen kann, wurde der Grenzfall kleiner $a$-Werte betrachtet. Es ergibt sich für ihn

$$
\sigma_{2 n}=1,6 \cdot 10^{-24} \mathrm{~cm}^{2} \quad \text { bei } E / \varepsilon=3 .
$$

Die bisher vorliegenden experimentellen Daten lassen für den Wirkungsquerschnitt $\sigma_{2 n}$ nur eine größenordnungsmäßige Abschätzung zu. Nach Rusinow ${ }^{18}$. liegt er ungefähr bei $10^{-25} \mathrm{~cm}^{2}$. $\mathrm{F}$ ü n f e r und $\mathrm{B}$ ot h e ${ }^{19}$ geben auf Grund neuerer Messungen an, daß er für Neutronen des Ra $\alpha+B e$

\begin{tabular}{|c|c|c|}
\hline \multirow{2}{*}{$\begin{array}{c}\text { Eínstrahlungs- } \\
\text { energie } E / \varepsilon\end{array}$} & \multicolumn{2}{|c|}{ W.Q. $\sigma_{2 n}\left(10^{-26} \mathrm{~cm}^{2}\right)$} \\
\hline & $\mathrm{I}$ & $\mathrm{II}$ \\
\hline 1,0 & 0 & 0 \\
1,4 & 0,322 & 0,141 \\
1,8 & 0,675 & 0,302 \\
2,2 & 0,890 & 0,405 \\
2,6 & 0,975 & 0,465 \\
3,0 & 0,990 & 0,477 \\
\hline
\end{tabular}

Tab. 4. Theoretische Wirkungsquerschnitte $\sigma_{2 n}$ für $a=2 \cdot 10^{-13} \mathrm{~cm}$ (Spalte I) und $a=3 \cdot 10^{-13} \mathrm{~cm}$ (Spalte II).

\begin{tabular}{|c|c|}
\hline $\begin{array}{c}\text { Kernradius } a \\
\left(10^{-13} \mathrm{~cm}\right)\end{array}$ & $\begin{array}{c}\text { W.Q. } \sigma_{2 n} \mathrm{für} E / \varepsilon=3 \\
\left(10^{-26} \mathrm{~cm}^{2}\right)\end{array}$ \\
\hline 1,5 & 1,55 \\
2,0 & 0,99 \\
25 & 0,66 \\
3,0 & 0,48 \\
3,5 & 0,37 \\
4,0 & 0,32 \\
\hline
\end{tabular}

Tab. 5. Theoretische Wirkungsquerschnitte $\sigma_{2 n}$ für $E / \varepsilon=3$ in Abhängigkeit von $a$.

sicher kleiner als $10^{-24} \mathrm{~cm}^{2}$ ist und $3 \cdot 10^{-25} \mathrm{~cm}^{2}$ nahekommen dürfte. Diese Werte sind größer als die hier berechneten. Doch handelt es sich bei diesen Messungen um den Mittelwert über ein Neutronenspektrum, das sich über einen größeren Energiebereich erstreckt, als die hier durchgeführte Rechnung zuläßt. Eine endgültige Entscheidung, ob die theoretischen Werte zu klein sind, kann danach noch nicht getroffen werden.

- Messungen von F. Houterman s ${ }^{26}$, nach denen der Mittelwert des Wirkungsquerschnitts über das Neutronenspektrum des $\mathrm{Po} \alpha+\mathrm{Be}$ $3,6 \cdot 10^{-24} \mathrm{~cm}^{2}$ beträgt, legen aber diese Vermutung nahe. Jedoch ist zu bedenken, daß der Prozeß, bei dem in einem Akt Neutronen und $\alpha$-Teilchen emittiert werden und der nicht getrennt beobachtet wird, eine ausschlaggebende Rolle spielen kann.

Die vorliegende Arbeit wurde am Institut für theoretische Physik der Universität Göttingen ausgeführt. Die Anregung stammt von Hrn. Prof. Dr. S. F lü g ge, dem ich für sein förderndes Interesse am Fortgang der Arbeit meinen herzlichen Dank aussprechen möchte. Für wertvolle Diskussionen und das entgegengebrachte Interesse danke ich ferner den HHrn. Prof. Dr. R. B e cke r, Prof. Dr. F. Ho u t e r mans und Dr. H. M a r s chall.

18 L. I. R u si now, Physik. Z. Sowjetunion 10, 219 [1936].

${ }_{19}$ E. Fü n f e r u. W. B ot he, Z. Physik 122, 769 [1944].

${ }_{20}$ F. H o u t e r m a n s, Nachr. Ges. Wiss. Göttingen, Math.-physik. Kil. 1946, 52. 